\title{
Tuning the BODIPY core for its potential use in DSSC: a quantum chemical approach
}

\author{
NARENDRA NATH GHOSH ${ }^{1}$, Md HABIB ${ }^{1}$, ANUP PRAMANIK ${ }^{2}$, PRANAB SARKAR ${ }^{2}$ and \\ SOUGATA PAL ${ }^{1, *}$ \\ ${ }^{1}$ Department of Chemistry, University of Gour Banga, Malda 732103, India \\ ${ }^{2}$ Department of Chemistry, Visva-Bharati University, Santiniketan 731235, India \\ *Author for correspondence (sougata@ugb.ac.in, sougatapal_1979@yahoo.co.in)
}

MS received 14 July 2017; accepted 23 August 2017; published online 30 March 2018

\begin{abstract}
Boron dipyrromethene (BODIPY) is a highly promising candidate for use in dye-sensitized solar cell (DSSC), because of its attractive absorption characteristics such as strong extinction coefficients in the visible and near-IR ranges (70000-80000 $\mathrm{M}^{-1} \mathrm{~cm}^{-1}$ ), large quantum yields, longer excited-state lifetime and also high solubility in many organic solvents. Moreover, the absorption peaks can be shifted towards longer wavelengths when functionalized at suitable positions of the BODIPY core. Herein, on the basis of density functional theory (DFT) and time-dependent DFT, we provide the opto-electronic properties of BODIPY core-functionalized dyes to see their applicability in organic DSSC. Our systematic analyses reveal that the 2,6 substituted dyes show better photovoltaic properties compared to the 3,5 substituted ones. On the basis of empirical relationship, we have also calculated the photo-induced electron injection times of some dye-TiO composites, which seem to be in the ultrafast time scale, thus rendering them a promising candidate for DSSC applications. Our theoretical studies provide that judiciously designed BODIPY core-derived dyes show certain unique spectroscopic and electronic features that make them highly advantageous in DSSC applications as compared to other organic dyes.
\end{abstract}

Keywords. BODIPY; DFT study; DSSC application.

\section{Introduction}

Dye-sensitized solar cells (DSSCs) are the most innovative artificial devices that mimic photosynthesis, which occurs in plants. Owing to the tunable electronic properties and low-cost availability of the constituent dyes, considerable scientific and industrial interests were paid to them as a potential candidate for source of renewable energy. Ruthenium polypyridyl complexes, in this regard, have received particular interest due to their impressive charge conversion efficiencies $(\eta>11 \%)$ under standard illuminating condition [1-3]. Despite of this high efficiencies, potential application of Ruthenium complexes in DSSCs is restricted owing to their relatively cost effectiveness and most importantly they are environmentally hazardous. During the past two decades, various attempts were made with different metal-free organic dyes with architectures donor-( $\pi$-spacer $)$-acceptor $(D-\pi-A)$ [4-6], D-A-D [7-10], etc. in the hope to find and replacement for metal complex dyes. Carbazole $(\eta=7.7 \%)$ [11,12], fluorene $(\eta=8.3 \%)[13,14]$, indoline $(\eta=8.0 \%)[15,16]$, phenothiazine $(\eta=6.22 \%)[17,18]$, due to their strong electron-donating abilities as well as efficient intermolecular charge transfer characteristics, are found to be efficient dyes in the $D-\pi-\mathrm{A}$ series. When $\pi$-spacer is concerned, the pyrrole moiety draws a particular attention as they could provide better excited state charge separation of the dyes arising due to less interactions with their neighbouring aromatic units [19]. Additionally, a vinyl group attached with those dyes can offer a more flexible polymer chain that improves the solubility in comparison to the directly-coupled aryl-aryl conjugated polymers [20].

Efficient use of a dye in the DSSC requires that the absorption spectra should cover a broad region of solar spectrum, i.e., the dye must have suitable band gap, while maintaining the frontier energy levels such that it can facilitate electron injection to a suitable semiconductor surface and it can prevent electron-hole recombination as well. In this regard, the use of 4,4-difluoro-4-bora-3a,4a-diaza-s-indacene, widely known as BODIPY dyes, provide high incident photon current efficiency (IPCE) for DSSCs. Adjustable structures of BODIPY dyes allow them to absorb all ranges of frequencies within the visible range extending to near-IR $[21,22]$. They possess extraordinary chemical and photochemical stabilities and also high solubility in many organic solvents. Moreover, the properties like fluorescence and redox can be tuned through the introduction of electron-donating and electron-withdrawing 
groups onto the core structure of the BODIPY [23-25]. To tune their optical properties, a variety of strategies were followed; some of them are functionalization of BODIPY core at meso- and 3,5-positions, fusion of aromatic ring into the core and replacement of the $\mathrm{C}$ atom with a $\mathrm{N}$ at 8 th position to form aza-derivatives, etc. [26-29]. BODIPY dyes exhibit a characteristic asymmetric charge redistribution, while undergoing $\mathrm{S}_{0}-\mathrm{S}_{1}$ transition upon excitation and thus, substitutions on different positions of BODIPY core have significant effect on its spectroscopic and electronic properties [30]. Very recently, 2,6-substituted BODIPY dyes have gained considerable attention in light harvesting devices because of their extended absorption characteristics in comparison to their mother components [27,28,31]. However, practical implementation of these materials as sensitizing agent is in its infancy and the reported efficiencies are still very low. Fukuzumi et al [32] reported the optoelectronic properties of BODIPY dyes indicating their photovoltaic performances in DSSCs using $\mathrm{TiO}_{2}$ as photo anode. Various research groups have designed and synthesized series of donor-acceptor-substituted BODIPY dyes with triphenylamines as donors and carboxylic or cyanoacrylic acids as acceptor counterpart [21,22,33].

To provide a new direction, further fundamental studies regarding the molecular structure, mode of substitution, optoelectronic properties and also the orientation and arrangement of the BODIPY dyes over the semiconductor surface are necessary. Detailed atomistic characterizations of these particular sensitizers are not yet explored sufficiently. Such an atomistic characterization not only provides information that complements the experimental work, but also helps to understand the structural and electronic properties for the design of novel sensitizers with improved performances. But, a systematic study on the computational characterization of the BODIPY dyes as a sensitizer for DSSCs is still scarce. Herein, we present a theoretical characterization of BODIPYbased sensitizers. Molecular geometry, electronic structure and spectral property of the sensitizers are investigated in the gas phase with the help of density functional theory (DFT) and time-dependent DFT (TD-DFT) calculations. Finally, photoinduced charge transfer (CT) abilities of the dyes were evaluated by means of some CT parameters, which in turn make a comparison among the studied dyes and thus, enlighten the superiority of a particular dye over the others. With this aim, we have also studied some composite systems consisting the dyes and a particular $\mathrm{TiO}_{2}$ nanocluster.

\section{Model and computation}

All the dyes are derived from the BODIPY core by functionalization particularly at 2,6 and 3,5 positions keeping the core as an acceptor of the typical D- $\pi-\mathrm{A}$ architecture. We choose four different types of donors, namely fluorene (FLU), carbazole (CAR), phenyl indoline (INDN) and phenothiazine (PTH) and their subsequent addition to the BODIPY core via $\pi$ linker results in different dyes as schematically shown in figure 1 . The vinyl pyrrole moiety as a $\pi$-bridge is introduced to fine tune the planar molecular configuration of the dyes and at the same time, it broadens the absorption spectra as already mentioned. To anchor the proposed dyes onto the semiconductor surface, cyanoacrylic acid was chosen as an anchoring group due its good electron-accepting and strongbinding abilities [34]. The effect of substitution of the donor groups was analysed in 2 and 3 positions in the BODIPY backbone keeping, cyanoacrylic acid as linker in 6 and 5 positions, respectively. Hereafter, we refer as Donor-2,6-BOD or 2,6 system and Donor-3,5-BOD or 3,5 systems.

The ground-state geometries of the dyes were optimized in the gas phase using DFT [35] with the B3LYP [36] hybrid functional. We have utilized 6-31G(d) basis function for computing the geometry and electronic structure of the BODIPY-based dyes. The use of B3LYP functional along with the $6-31 \mathrm{G}(\mathrm{d})$ basis sets for predicting accurate geometry and electronic structure for such small organic molecules is well tested and reported in the literature [21,37]. The excitation energies of the dyes for the lowest 20 singlet-singlet transitions were obtained by performing TD-DFT calculations on the respective ground-state geometries using the same basis sets. We employed the polarizable continuum model (PCM) [38] using the integral equation formalism variant (IEFPCM) to account for the solvent effect, while computing the absorption spectra of the dye in methanol. In the view of a practical donor-acceptor model, we choose $\left(\mathrm{TiO}_{2}\right)_{15}$ cluster as an electron accepting material. It is noteworthy that the use of such cluster model for representing semiconductor surface is being successful for predicting photovoltaic performance of different nanocomposites, especially for DSSCs $[39,40]$. The selected dyes are anchored onto the $\left(\mathrm{TiO}_{2}\right)_{15}$ surface and the composite systems are optimized with the same exchange correlation functional. For the basis sets, we used 6-31G(d) for all the atoms except Ti for which LANL2DZ basis with LANL2 pseudopotential was used [41]. All types of calculations were done using the Gaussian 09 program packages [42]. Adsorption energies $\left(E_{\text {ads }}\right)$ of the dyes on $\left(\mathrm{TiO}_{2}\right)_{15}$ cluster were obtained by equation (1):

$$
E_{\text {ads }}=E_{\mathrm{TiO}_{2}+\text { dye }}-\left(E_{\mathrm{TiO}_{2}}+E_{\text {dye }}\right),
$$

where $E_{\mathrm{TiO}_{2}}, E_{\text {dye }}$ and $E_{\mathrm{TiO}_{2}+\text { dye }}$ are the ground-state energies at optimized geometries of the cluster, dye and dyesemiconductor heterostructures, respectively.

The electron injection time ( $\tau_{\text {inj }}$, in femtosecond) from the dye to $\mathrm{TiO}_{2}$ was estimated by a model derived from the Newns-Anderson approach;

$$
\tau_{\mathrm{inj}}=\frac{658}{\Gamma},
$$

where $\tau_{\text {inj }}$ is inversely proportional to the broadening ( $\Gamma$, in $\mathrm{meV}$ ) of the donor orbital of the dye upon adsorption. 

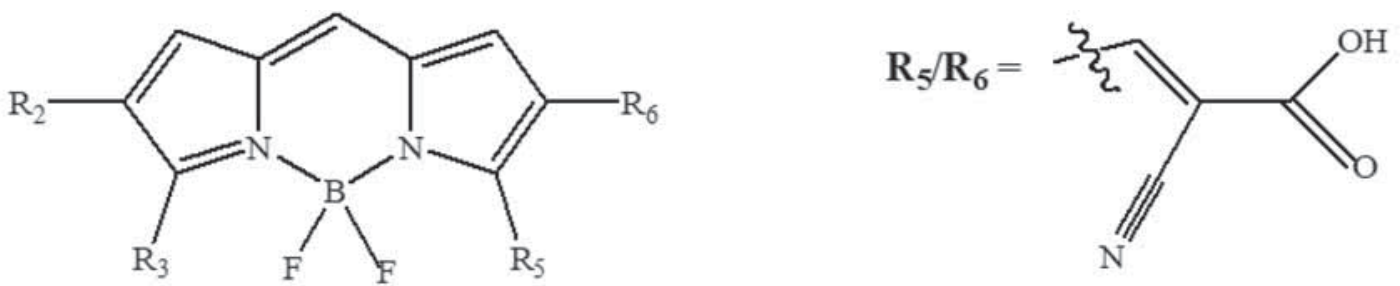

\section{BOD}

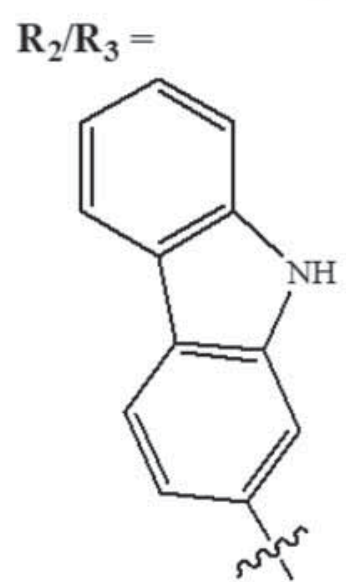

CAR-2,6-BOD CAR-3,5-BOD

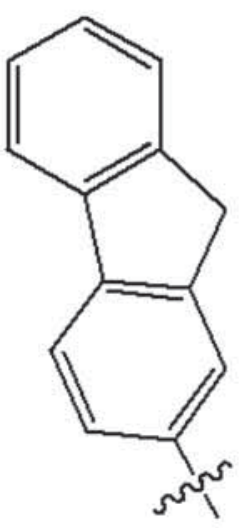

FLU-2,6-BOD FLU-3,5-BOD

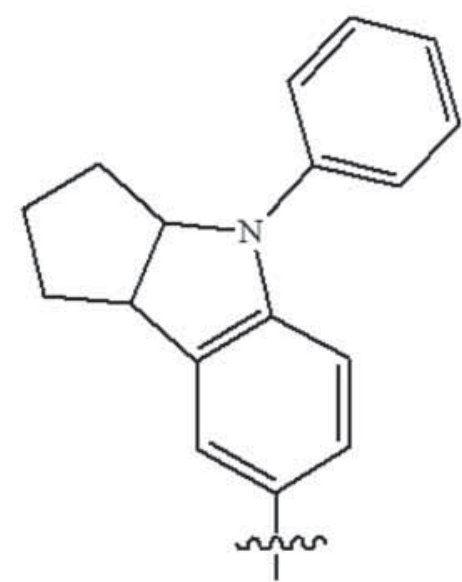

INDN-2,6-BOD INDN-3,5-BOD

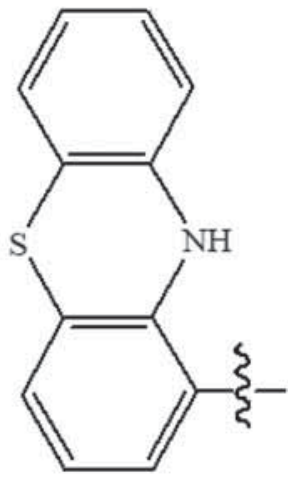

PTH-2,6-BOD PTH-3,5-BOD

Figure 1. Schematic representation of basic units of the studied dyes. The binding sites are shown by unsaturations with wavy bonds.

\section{Results and discussion}

\subsection{Electronic energy level and molecular orbital analysis}

Sensitizer is a key component of DSSC, and always desires to be low band gap with very specific energy levels [43]. The energies of HOMO (H) and LUMO (L) orbitals can be used as a simple test of the electronic properties around the fundamental band gap region of semiconductor. The calculated band gap values of the sensitizers are tabulated in table 1 . It is revealed that 2,6 dyes always yield lower band gap compared to that of 3,5 dyes, and it can be attributed by the fact that resonance effect is much prominent with the former than the latter. Band gap values of the proposed dyes oscillate within a narrow region, in between $1.60-1.73$ and $1.74-1.88 \mathrm{eV}$ for 2,6 and 3,5 systems, respectively. The INDN and PTH donors always have a tendency to reduce such gap and interestingly the dyes derived from them, namely INDN-2,6-BOD and PTH-2,6BOD are found to be iso-band gap dyes. Most importantly, every donor group has almost same effect towards altering the band gap for a particular system. Figure 2 represents the energy level alignment of HOMO and LUMO of the dyes, with respect to the conduction band of $\mathrm{TiO}_{2}$ situated at $4.0 \mathrm{eV}$ [44]. From the figure, it is expected that all the studied dyes will offer type II band alignment, while coupled with
$\mathrm{TiO}_{2}$ surface, however, a little band bending may occur due to charge transfer interaction between the dye and $\mathrm{TiO}_{2}$ surface. Note that in type II band alignment, the isosurfaces of HOMO and LUMO of the composite system lie on two different constituents. To have a spontaneous charge transfer from the dye to $\mathrm{TiO}_{2}$, LUMO of dye should be located right above the conduction band of $\mathrm{TiO}_{2}(4.0 \mathrm{eV})$. Moreover, for effective dye regeneration, $\mathrm{HOMO}$ of the dye should lie below the reduction potential energy of the $\mathrm{I}_{3}^{-} / \mathrm{I}^{-}$electrolyte $(4.80 \mathrm{eV})$ [44]. So, from the relative band energy alignment as shown in figure 2 , it is expected that the BODIPY dyes will serve the role of a good sensitizer.

We have performed molecular orbital analysis to understand the molecular structure and the associated electronic distribution within the dyes. In figure 3, we display selected spatial molecular distribution of the designed dyes. At a glance, it is noticeable that 2,6 dyes offer better charge separation than 3,5 dyes. Among 2,6 dyes, such spatial separation is prominent in the case of INDN and PTH donor dyes. One can see that the electron densities of HOMO for INDN2,6-BOD, PTH-2,6-BOD are located over the electron-rich donor moiety, while LUMO densities are more concentrated over the acceptor (BODIPY) as well as anchoring group. This fact is being confirmed upon fragment analysis as shown in supplementary table S1. The contribution 
Table 1. Estimated HOMO-LUMO gap $\left(E_{\mathrm{HL}}\right)$, absorption maxima $\left(\lambda_{\max }\right)$ and oscillator strength $(f)$ with major transition composition of the dyes.

\begin{tabular}{|c|c|c|c|c|c|}
\hline Dye & $E_{\mathrm{HL}}(\mathrm{eV})$ & $\lambda_{\max }(\mathrm{nm})$ & Transition & $f$ & Major composition \\
\hline \multirow[t]{2}{*}{ FLU-2,6-BOD } & \multirow[t]{2}{*}{1.73} & 390 & $\mathrm{~S}_{0}-\mathrm{S}_{6}$ & 0.973 & $\mathrm{H} \rightarrow \mathrm{L}+2(69 \%)$ \\
\hline & & 800 & $\mathrm{~S}_{0}-\mathrm{S}_{1}$ & 0.548 & $\mathrm{H} \rightarrow \mathrm{L}(100 \%)$ \\
\hline \multirow[t]{2}{*}{ CAR-2,6-BOD } & \multirow[t]{2}{*}{1.71} & 386 & $\mathrm{~S}_{0}-\mathrm{S}_{6}$ & 1.052 & $\mathrm{H} \rightarrow \mathrm{L}+2(68 \%)$ \\
\hline & & 806 & $\mathrm{~S}_{0}-\mathrm{S}_{1}$ & 0.547 & $\mathrm{H} \rightarrow \mathrm{L}(100 \%)$ \\
\hline \multirow[t]{2}{*}{ INDN-2,6-BOD } & \multirow[t]{2}{*}{1.60} & 387 & $\mathrm{~S}_{0}-\mathrm{S}_{5}$ & 1.086 & $\mathrm{H} \rightarrow \mathrm{L}+2(68 \%)$ \\
\hline & & 863 & $\mathrm{~S}_{0}-\mathrm{S}_{1}$ & 0.526 & $\mathrm{H} \rightarrow \mathrm{L}(100 \%)$ \\
\hline \multirow[t]{2}{*}{ PTH-2,6-BOD } & \multirow[t]{2}{*}{1.60} & 437 & $\mathrm{~S}_{0}-\mathrm{S}_{5}$ & 0.624 & $\mathrm{H}-3 \rightarrow \mathrm{L}(78 \%)$ \\
\hline & & 882 & $\mathrm{~S}_{0}-\mathrm{S}_{1}$ & 0.285 & $\mathrm{H} \rightarrow \mathrm{L}(100 \%)$ \\
\hline \multirow[t]{2}{*}{ FLU-3,5-BOD } & \multirow[t]{2}{*}{1.88} & 671 & $\mathrm{~S}_{0}-\mathrm{S}_{1}$ & 1.274 & $\mathrm{H} \rightarrow \mathrm{L}(100 \%)$ \\
\hline & & 379 & $\mathrm{~S}_{0}-\mathrm{S}_{5}$ & 0.385 & $\mathrm{H} \rightarrow \mathrm{L}+1(46 \%)$ \\
\hline \multirow[t]{2}{*}{ CAR-3,5-BOD } & \multirow[t]{2}{*}{1.87} & 673 & $\mathrm{~S}_{0}-\mathrm{S}_{1}$ & 1.296 & $\mathrm{H} \rightarrow \mathrm{L}(100 \%)$ \\
\hline & & 376 & $\mathrm{~S}_{0}-\mathrm{S}_{6}$ & 0.436 & $\mathrm{H} \rightarrow \mathrm{L}+1(53 \%)$ \\
\hline \multirow[t]{2}{*}{ INDN-3,5-BOD } & \multirow[t]{2}{*}{1.76} & 721 & $\mathrm{~S}_{0}-\mathrm{S}_{1}$ & 1.130 & $\mathrm{H} \rightarrow \mathrm{L}(100 \%)$ \\
\hline & & 382 & $\mathrm{~S}_{0}-\mathrm{S}_{5}$ & 0.304 & $\mathrm{H} \rightarrow \mathrm{L}+1(44 \%)$ \\
\hline \multirow[t]{2}{*}{ PTH-3,5-BOD } & \multirow[t]{2}{*}{1.74} & 604 & $\mathrm{~S}_{0}-\mathrm{S}_{2}$ & 0.965 & $\mathrm{H}-1 \rightarrow \mathrm{L}(93 \%)$ \\
\hline & & 364 & $\mathrm{~S}_{0}-\mathrm{S}_{9}$ & 0.218 & $\mathrm{H}-1 \rightarrow \mathrm{L}+1(44 \%)$ \\
\hline
\end{tabular}

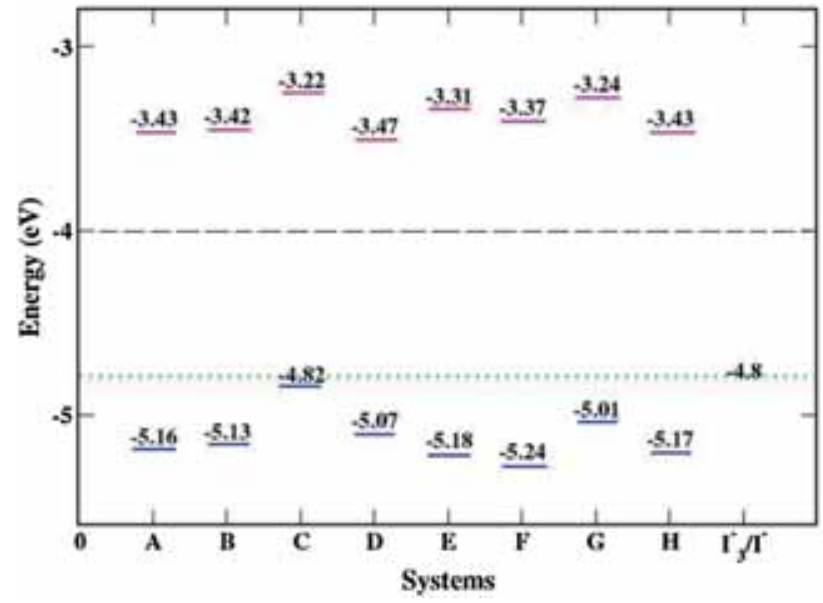

Figure 2. Alignment of frontier energy levels of the dyes; horizontal lines with magenta and blue colours represent LUMO and HOMO levels, respectively. Dotted line indicates standard potential of the $\mathrm{I}_{3}^{-} / \mathrm{I}^{-}$redox couple. A: FLU-2,6-BOD; B: CAR-2,6-BOD; C: INDN-2,6-BOD; D: PTH-2,6-BOD; E: FLU-3,5-BOD; F: CAR3,5-BOD; G: INDN-3,5-BOD and H: PTH-3,5-BOD.

of donor towards HOMO is almost double for PTH, while comparing the same with other donor groups. The following trends of increasing degree of charge separation; FLU $<$ CAR $<$ INDN $<$ PTH is clearly observed. Obviously, it is well characteristics of good sensitizer which leads to facile electron transfer [45]. But, unexpectedly such spatial separations are not so prominent for 3,5 dyes as compared to the 2,6 ones. So, we may conclude that the 2,6 dye are more resistive towards recombination of photogenerated charge carriers, thereby leading them as superior candidates for DSSC.

\subsection{Optical properties}

Maximum absorption coverage of electromagnetic radiation is the primary focus for an effective sensitizer and hence, we proceed to investigate the optical properties of our designed sensitizers. Calculated absorption maxima, oscillator strength $(f)$ and corresponding major transition character in vacuum of the designed dyes are presented in table 2, while simulated absorption spectrum is displayed in figure 4. Broad UV-Vis spectra of all the dyes ranging from UV to IR region indicate that they are capable of harvesting broad spectrum of solar radiation. All the 2,6 dyes show sharp peaks around 350$400 \mathrm{~nm}$ (figure 4, left panel), which is mainly due to the $\pi-\pi^{*}$ type of transition. Transition from HOMO to some upper unoccupied levels is responsible for this peak. Another lower intensity peak appearing near-NIR region at 800-880 $\mathrm{nm}$ corresponds to the band gap absorption (transition from HOMO to LUMO level). Noteworthy is that these absorption bands are quite sensitive towards donor group and a bathochromic shift is noticed when going from FLU to PTH donor. Furthermore, significant red shifting of absorption maxima is also observed when we simulate the spectra in polar solvent like methanol (see supplementary table S2). On the other hand, 3,5 substituted dyes show broader spectrum (figure 4, right panel) than that of 2,6 substituted dyes as also evidenced from the experimental findings [46]. But the scenario of electronic transition type is completely reversed as compared to the 2,6 dye systems. Here, the HOMO-LUMO transition band enters into the visible region (between 550 and $800 \mathrm{~nm}$ ) with enhanced intensity, while the high energy 


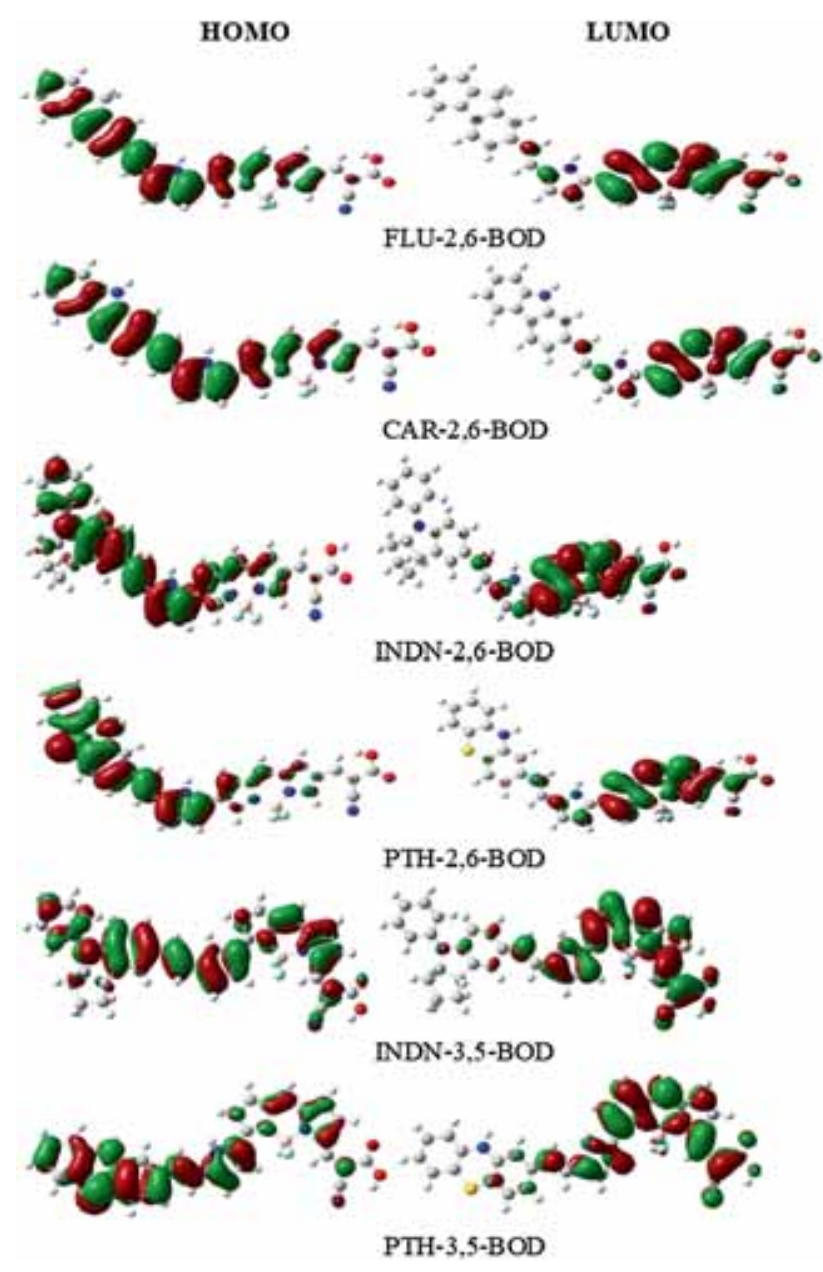

Figure 3. Optimized structure with HOMO and LUMO electron density distribution patterns of some selected dyes in gas phase.

band becomes relatively weaker in comparison with the 2,6 substituted dyes.

\subsection{Charge separation indices}

As already discussed, photo-induced electronic excitation and subsequent spatial charge separation is the key to accelerate charge transfer processes. To examine the intramolecular charge transfer from one part of the dye to the other part, we present charge density difference for the 2,6 sensitizers using Multiwfn program package [47]. The electron density difference associated with HOMO-LUMO transition is shown in figure 5 (left panel), where cyan and pink colours correspond to zone of electron density depletion and increment, respectively. It is evident that all the systems show distinct charge separation where significant electron density difference is observed between the donor and acceptor regions. Obviously, this plot is for qualitative assessment purpose and it is quite hard to compare the extent of charge separation, hence, we need more accurate and quantitative evaluation.
Table 2. Calculated driving force ( $\Delta G_{\text {inj }}$ in eV), excited state life time ( $\tau$ in ns), light harvesting efficiency (LHE) and open circuit voltage $\left(e V_{\mathrm{OC}}\right.$ in $\left.\mathrm{eV}\right)$ of the dyes.

\begin{tabular}{lccccc}
\hline Dye & $E_{\text {dye }}^{*}$ & $\Delta G_{\text {inj }}$ & $\tau(\mathrm{ns})$ & LHE & $e V_{\text {OC }}$ \\
\hline FLU-26-BOD & 1.98 & 2.02 & 17.5 & 0.893 & 0.47 \\
CAR-26-BOD & 1.92 & 2.08 & 17.7 & 0.911 & 0.48 \\
INDN-26-BOD & 1.62 & 2.38 & 21.3 & 0.918 & 0.78 \\
PTH-26-BOD & 2.23 & 1.77 & 40.1 & 0.762 & 0.43 \\
FLU-35-BOD & 3.33 & 0.67 & 5.3 & 0.946 & 0.70 \\
CAR-35-BOD & 3.40 & 0.60 & 5.20 & 0.949 & 0.63 \\
INDN-35-BOD & 3.28 & 0.72 & 6.9 & 0.926 & 0.76 \\
PTH-35-BOD & 3.12 & 0.88 & 21.5 & 0.891 & 0.43 \\
\hline
\end{tabular}

Spatial overlap $(\Lambda)$, which directly reflects on number of overlap zones among electrons and holes upon photo excitation. In figure 5 (right panel), the typical green colour is the representation of such overlap density. We note the descending trend of overlap: FLU > CAR > INDN > PTH. The diagnostic lowest value of it for PTH donor containing sensitizer makes it an appealing candidate for charge separation. $d_{\text {CT }}$ defines the spatial distance between the two barycenters of the density depletion and enhancement upon excitation, which can give a quantitative estimation of the charge transfer length. We find that $d_{\mathrm{CT}}$ is dependent on the donor group and the trend of $d_{\mathrm{CT}}$ follows the order: FLU $<\mathrm{CAR}<\mathrm{INDN}$ $<$ PTH. Generally, larger value of charge transfer length is a diagnostic of prominent charge separation, which in turn indicates facile charge transfer to a suitably aligned semiconductor anode surface. Large $d_{\mathrm{CT}}$ is also indicative of slower charge recombination [48,49]. Now, if we define $H$ as the half of the sum of the root-mean-square distribution of the density depletion and enhancement zone, then $t$ is the difference between $d_{\mathrm{CT}}$ and $H$ is the degree of separation between the two zones. In other words, $t$ evaluates the through space charge transfer character upon the electronic excitation. It should be pointed out that the calculated $t$ values gradually increases from FLU-2,6-BOD to PTH-2,6-BOD as shown in figure 5. Combining all these $C T$ indices, we can conclude that the dye PTH-26-BOD followed by INDN-26-BOD should be ideal for solar cell component.

\subsection{Light harvesting properties}

The absorption coverage of dye within the solar irradiation spectrum exerts a crucial influence on the short circuit current $J_{\mathrm{SC}}$, one of the most important factors governing efficiency of a solar cell device. It is simply a function of light harvesting efficiency (LHE), which can be expressed as LHE $=1-10^{-f}$, where $(f)$ is the oscillating strength of the dye [50]. From table 2, it is observed that calculated LHE are in the order of around 0.9 for all the systems and possibly high value of short circuit current $\left(J_{\mathrm{SC}}\right)$ can be achieve with these dyes. It is necessary to mention that the LHE values 

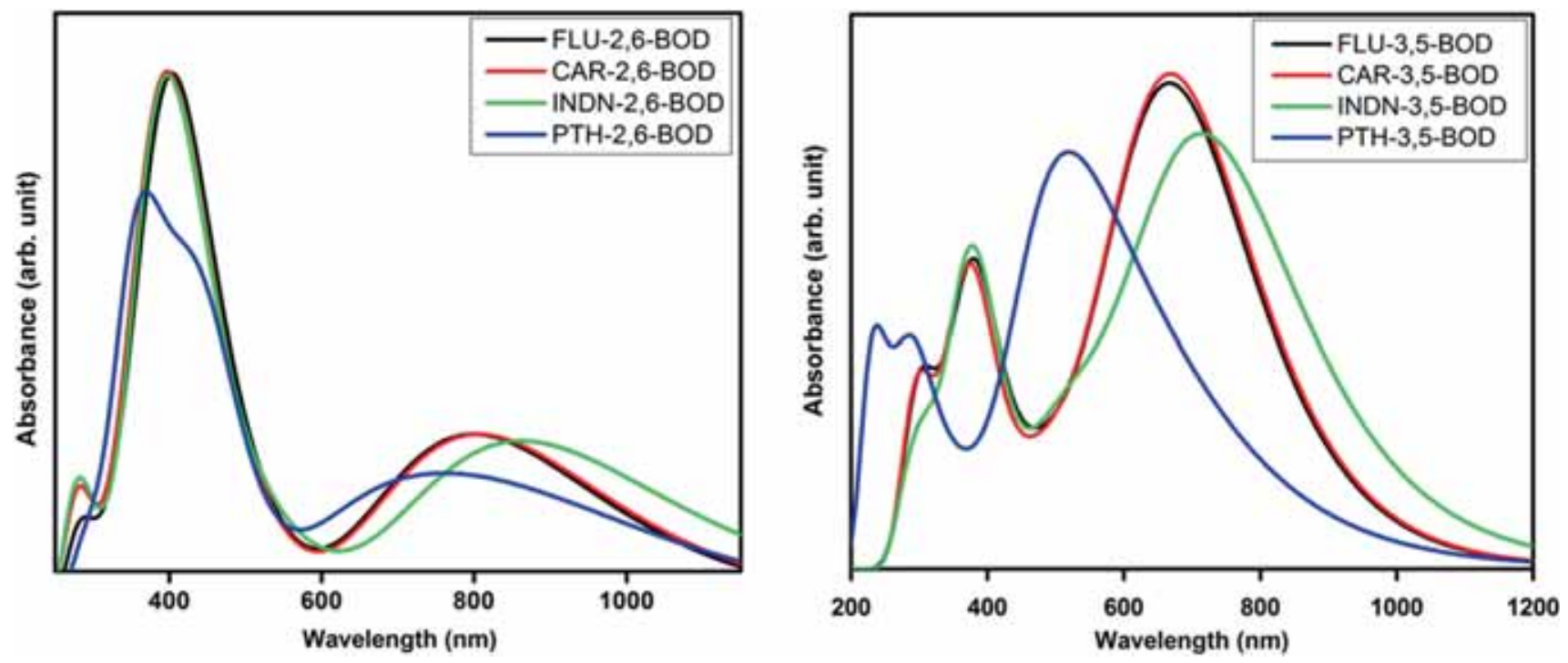

Figure 4. Calculated photo-absorption spectra of the dyes in vacuum, left and right panels for 2,6 and 2,5 dyes, respectively.

for 3,5 dyes are slightly higher than corresponding 2,6 dyes. Among 2,6 systems, INDN achieves the maximum value of LHE and hence, we expect the highest $J_{\mathrm{SC}}$ value for it. Open circuit voltage $\left(V_{\mathrm{oc}}\right)$ is another important parameter to evaluate overall efficiency of a solar cell which can be simplified as the energy difference between the LUMO of the dye $\left(E_{\mathrm{LUMO}}\right)$ and the conduction band of the semiconductor surface $\left(E_{\mathrm{CB}}\right)$ [43]. For an efficient solar cell, high value of $V_{\mathrm{oc}}$ is always desirable [51]. A similar trend like LHE is observed (table 2) when we compare positioned dependency of $e V_{\text {oc }}$. Once more, we find INDN occupying the highest place in this trend.

\subsection{Driving force of electron injection and excited state lifetime}

In this part, we will focus on excited state properties of the designed sensitizers. We have calculated driving force of electron injection $\left(\Delta G_{\text {inj }}\right)$ from the excited dye to the conduction band of the semiconductor. Perhaps it is the most important photovoltaic parameter which is directly related to $J_{\mathrm{SC}}$ value and provides an idea about the rate of electron transfer. Recent studies indicate that interfacial electron transfer rate can be evaluated by driving force $\left(\Delta G_{\mathrm{inj}}\right)$ [52]. According to the Marcus theory, larger the value of driving force $\left(\Delta G_{\text {inj }}\right)$, faster the rate of electron injection. The free energy change (in $\mathrm{eV}$ ) for the electron injection process can be expressed as $\Delta G_{\text {inj }}=E_{\text {dye }}^{*}-E_{\text {Св }}$. The results of our quantum chemical calculations are summarized in table 2 . The negative value of $\Delta G_{\text {inj }}$ establishes the described phenomenon of spontaneous charge transfer from the dyes to the semiconductor. From the tabulated data, it is seen that (i) 2,6 substituted dyes have higher value of $\Delta G_{\text {inj }}$, almost three times greater than corresponding 3,5 dyes; (ii) the driving force is higher for INDN than the other dyes in 2,6 systems and hence, we may expect rapid interfacial electron transfer for this system.

The decay of excited state $\left(S_{1}\right)$ to ground state $\left(S_{0}\right)$ is an important competing process with the electron injection. Excited state(s) lifetime ( $\tau$ in nanosecond), which is one of the leading factors for charge transfer and hence, the overall efficiency of DSSC [53]. Higher the value of excited state lifetime, longer will be the stability of the dye in its cationic form and hence, one could expect a more facile electron transfer from the dye to the semiconductor surface. In this consequence, it is expected that to have a longer life span, the excited state should possess not only sufficient charge separation, but also long range distance between the opposite charge densities. The parameter of lifetimes was evaluated [53] using, $\tau=1.44 / f E^{2}$ where $E$ is the excitation energy for $\mathrm{S}_{0}-\mathrm{S}_{1}$ electronic states $\left(\mathrm{cm}^{-1}\right)$ and $f$ is oscillator strength. As indicated in the table 2, we can say that the tendency to remain longer time in the $S_{1}$ state is higher for 2,6 dyes when compared with 3,5 systems. Interestingly, we find the highest value (40 ns) for the dye comprising PTH donor of the 2,6 system, followed by INDN ( $21 \mathrm{~ns}$ ). Prominent long range charge separation and poor overlap density between electron and hole is attributed to the longer life of excited state for these sensitizers.

\subsection{Dye-semiconductor interface}

After having electronic as well as spectroscopic characterizations of all the studied dyes described earlier, we further proceed to simulate the performance of DSSC device in more realistic way. To do so, we first choose a $\left(\mathrm{TiO}_{2}\right)_{15}$ cluster with $\mathrm{C}_{2 \mathrm{~h}}$ symmetry since it shows bulk-like properties [54]. The cluster is a nice candidate for solar cell counterpart as described previously $[39,40]$. Strength of interaction between 

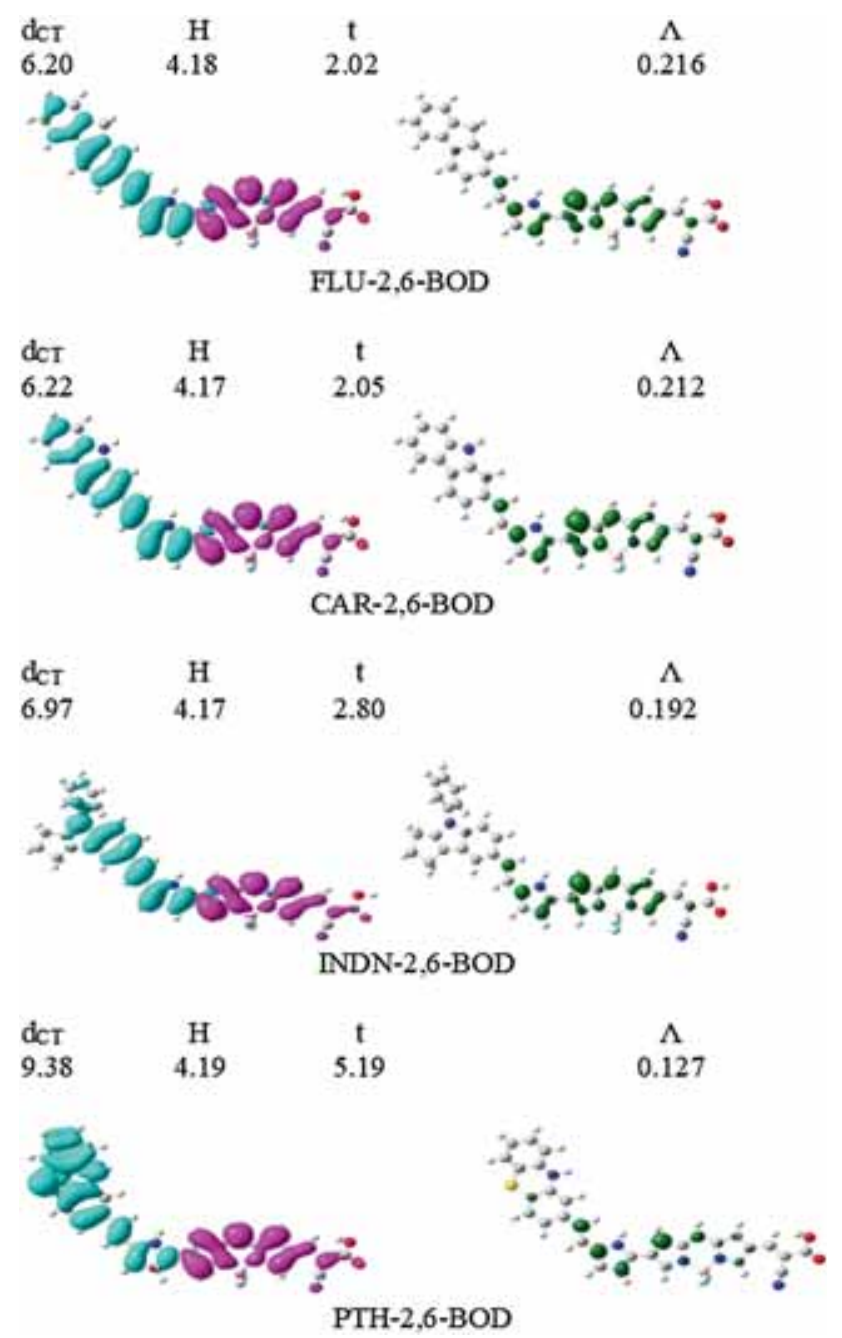

Figure 5. Left panel: electron density difference map for 2,6 system, where cyan and pink colours represent the zone of electron density depletion and increment, respectively. Right panel: extent of overlap among the density of electron and hole.

the dye and semiconductor is of crucial importance as it directly influences the adsorption energy of the composite system. As far as the suitability of the sensitizer is concerned, from the previous calculations, we selectively choose the INDN and PTH donor dyes for further investigation. They are coupled with the cluster and allowed to relax without symmetry constrained. As shown in supplementary figure $\mathrm{S} 1$, in the optimized structure, the dyes are aligned almost perpendicular to the $\mathrm{TiO}_{2}$ cluster surface, linked by two $\mathrm{O}$ atoms of the carboxylic acid bonded with one Ti atom in a bidentate-bridging adsorption mode. The calculated adsorption energy [55], $E_{\text {ads }}$ values for dyes@ $\mathrm{TiO}_{2}$ are in the range of $1.57-1.65 \mathrm{eV}$ (36-38 $\mathrm{kcal} \mathrm{mol}^{-1}$ ), indicating strong interactions between the dyes and the $\mathrm{TiO}_{2}$ surface. The negative value of adsorption energy confirms the process as exothermic in nature and binding motif is stable enough. Further, the frontier molecular orbital (FMO) distribution offers an intuitive view about charge separation. From figure 6, it is seen that HOMOs of the composites are exclusively localized on the donor part of the sensitizer; in contrast, LUMO orbitals are solely contributed by the semiconductor. Thus, from the spatial separation of electron and hole, one could expect a slower recombination dynamics for the nanocomposites after photoexcitations. We next proceed to compute the electronic excitations of the composites by TD-DFT method. The calculation was performed by taking first 10 lowest single-singlet transitions at the same level of theory as the energy minimization of the composites. For both heterointerfaces, we found a new absorption band near infrared region at around $950 \mathrm{~nm}$ (see table 3). Appearance of new peak for the dye $@ \mathrm{TiO}_{2}$ compared to the free dye clearly indicates that the systems undergo direct electronic transition (type II) as recently illustrated by Zarate et al [39]. $\mathrm{HOMO} \rightarrow \mathrm{LUMO}+n$ transition shows the major contribution to the maximum absorbance for both the systems. In figure 6 , we plot some selective levels to gain more insight on the photoinduced mechanism. For INDN@ $\mathrm{TiO}_{2}$, the electronic transition occurs near IR region from HOMO to LUMO+9, where the mixed isosurface (LUMO+9) are contributed by both dye and cluster. It should be pointed out that negligible amount of charge density is located over the anchoring moiety. On the other hand, for PTH@ $\mathrm{TiO}_{2}$, the electronic transition corresponds to linear combination of HOMO to LUMO+7 and HOMO to LUMO+8. It is noticeable that the contributing LUMO+7 and LUMO+8 orbitals are mainly arisen from the mixing of charge densities of both dye and the cluster. Moreover, the nature of charge density distribution is quite distinctive than the former that the anchor group also provides sufficient contribution towards the said higher LUMO levels. From the energetic point of view, in case of former, the electron jumps to the higher energy orbital (LUMO+9, $-3.42 \mathrm{eV}$ ), while such transition occurs at the deeper electronic levels (LUMO+7, $-3.62 \mathrm{eV}$ and LUMO+8, $-3.58 \mathrm{eV}$ ) for later system. Hence, we can argue that the excited electron transfer to the conduction band edge of $\mathrm{TiO}_{2}$ would be more facile for PTH@ $\mathrm{TiO}_{2}$.

\subsection{Photo-induced electron injection}

To understand the key ultrafast processes associated at the dye-semiconductor interface, we demonstrate our studies on the interfacial electron injection. The structure and the precise nature of excited state of the organic-inorganic conjugate determine the electron injection efficiency, in which the photoexcited electron injection occurs in the ultra fast time scale. To quantify this phenomenon, extensive dynamical simulation [56] is required, but for the time being, the purpose can be fulfilled though to some extent moderately, using NewnsAnderson model according to which electron injection time (in femto second) is inversely related to the band broadening [see equation (2)] [57-59]. The inter-state coupling between LUMO of the dye and the conduction band of the semiconductor should be strong for an efficient electron injection from the dye to SC surface. Our calculation reveals that both the 


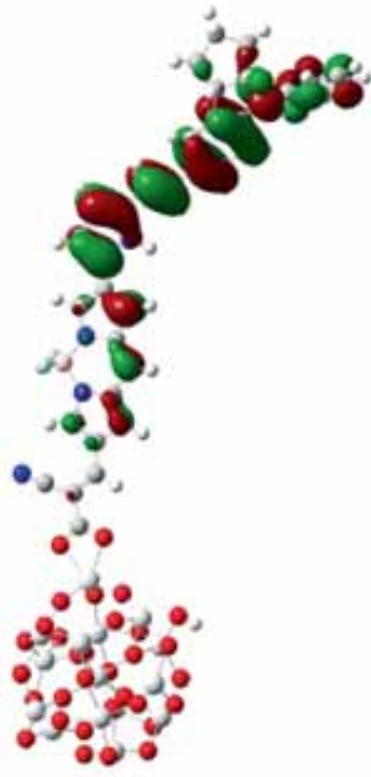

$\mathrm{H}$

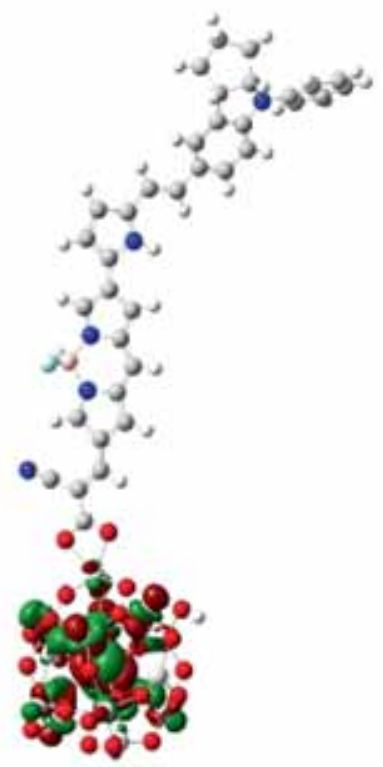

L

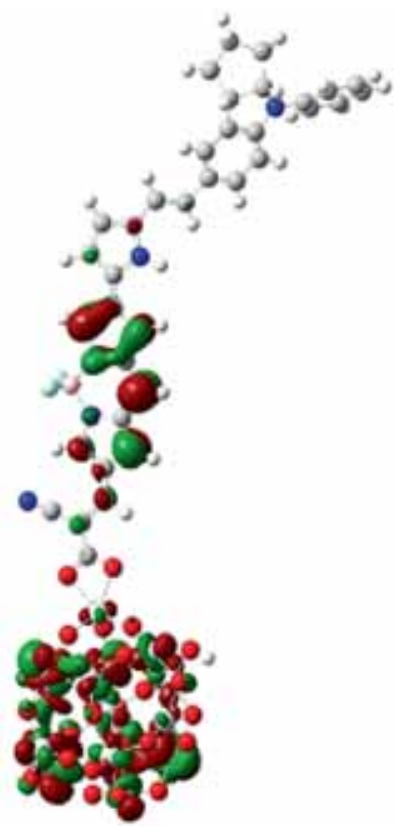

$\mathrm{L}+9$

\section{INDN@ $\mathrm{TiO}_{2}$}

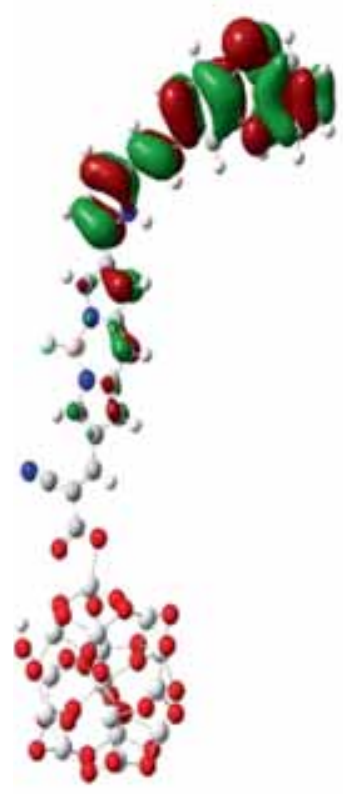

$\mathrm{H}$

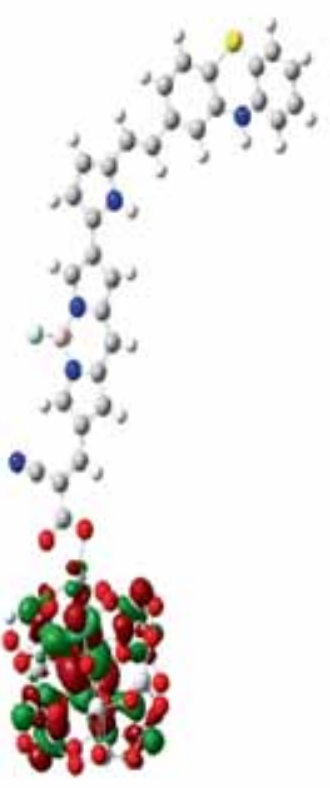

L

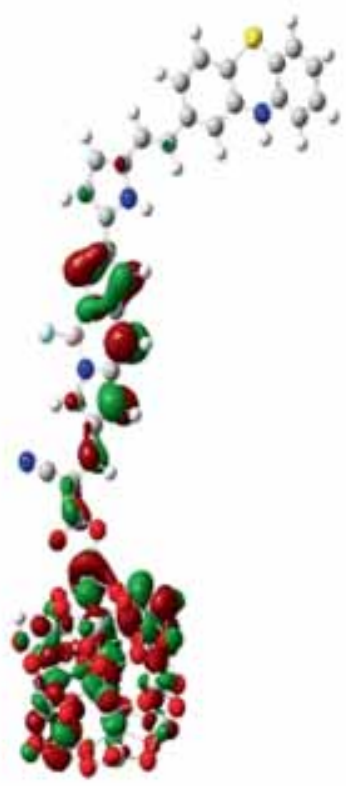

$\mathrm{L}+7$

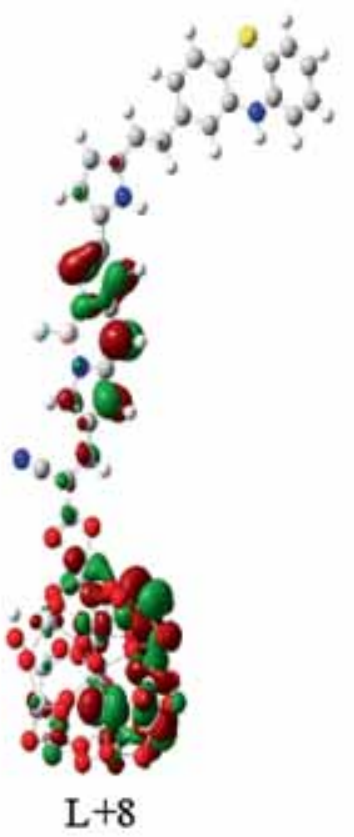

\section{$\mathrm{PTH} @ \mathrm{TiO}_{2}$}

Figure 6. Electron density distribution of some selective orbitals of the dye- $\mathrm{TiO}_{2}$ composites.

systems viz. INDN@ $\mathrm{TiO}_{2}$ (1.5 fs) and $\mathrm{PTH} @ \mathrm{TiO}_{2}(0.71 \mathrm{fs})$ show ultrafast electron injection. Although electron transfer rate is very comparable, but we are curious to know why the injection time for PTH@ $\mathrm{TiO}_{2}$ is faster? Upon photo excitation, electronic coupling and electron transfer take place between the dye's LUMO and conduction band of SC. Greater the coupling between the dye and semiconductor, greater is the band broadening and hence, faster will be the electron transfer rate as per the previous study by Wei et al [60]. On close inspection of LUMO levels of composite systems, we 
Table 3. Estimated adsorption energy $\left(E_{\text {ads }}\right)$, absorption maxima $\left(\lambda_{\max }\right)$ with transition character and electron injection time $\left(\tau_{\text {inj }}\right)$ of dye $@ \mathrm{TiO}_{2}$ composites.

\begin{tabular}{lcccc}
\hline System & $E_{\text {ads }}(\mathrm{eV})$ & $\lambda_{\max }(\mathrm{nm})$ & Transition characteristics & $\tau_{\text {inj }}(\mathrm{fs})$ \\
\hline${\mathrm{INDN} @ \mathrm{TiO}_{2}}-1.57$ & $963(0.188)$ & H-L+9 & 1.50 \\
$\mathrm{PTH} \mathrm{TiO}_{2}$ & -1.65 & $929(0.129)$ & $\mathrm{H}-\mathrm{L}+6, \mathrm{H}-\mathrm{L}+7, \mathrm{H}-\mathrm{L}+8$ & 0.71 \\
\hline
\end{tabular}

found mixing of dye's LUMO and conduction band of SC is of greater extent (see supplementary table S3) for ${\mathrm{PTH} @ \mathrm{TiO}_{2}}$ than its competitor. Such an ultrafast rate is deemed to be sufficient for an efficient electron injection.

\section{Conclusion}

We have applied computational techniques in combination with DFT and TDDFT methodologies to study different photovoltaic properties of BODIPY core stimulated designed dyes. First, we have studied the opto-electronic properties of the individual dyes. It was revealed that the core modifications via the positions viz. 2,6 or 3,5 have a significant role in altering the photovoltaic parameters, such as $V_{\mathrm{OC}}$, LHE, etc. and consequently on energy conversion efficiency. Effect of different donors also makes a valuable impact on such parameters. Electron-rich groups have played a vital role for narrowing band gap and leading to broad absorption coverage indicative of potential sensitizers in typical DSSC. Specifically, 2,6 dyes have superior photovoltaic properties when compared to 3,5 ones. Interestingly, among 2,6 systems, PTH-2,6-BOD and INDN-2,6-BOD show better photovoltaic properties than the others and indeed being iso-band gap value, they exhibit striking comparison among properties of interest. Thermodynamic driving force for of electron injection is remarkably sound for INDN-2,6-BOD, while charge separation is quite better for PTH-2,6-BOD. Regarding photoinduced electron injection, both of them show ultrafast phenomenon, however, faster injection occurs for PTH-2,6-BOD sensitizer. We then compute the similar photovoltaic properties of the more realistic dye- $\mathrm{TiO}_{2}$ composite systems. Our calculations demonstrate that PTH@ $\mathrm{TiO}_{2}$ composite system acts as a superior material for solar energy harvesting process. Finally, we strongly believe that our computational results will stimulate the experimental researchers and provide a guideline to them for designing and fabricating BODIPY-based photovoltaic devices.

\section{Acknowledgements}

We sincerely acknowledge UGC, New Delhi, Govt. of India, for partial financial support. The financial support from SERB-DST, New Delhi, through the Project Ref. No. CS-085/2014 is gratefully acknowledged.

\section{References}

[1] O'regan B and Grfitzeli M 1991 Nature 353737

[2] Nazeeruddin M K, Pechy P, Renouard T, Zakeeruddin S M, Humphry-Baker R, Comte P et al $2001 \mathrm{~J}$. Am. Chem. Soc. 123 1613

[3] Nazeeruddin M K, De Angelis F, Fantacci S, Selloni A, Viscardi G, Liska P et al 2005 J. Am. Chem. Soc. 12716835

[4] Mishra A, Fischer M K and Bäuerle P 2009 Angew. Chem. Int. Ed. 482474

[5] Wu Y and Zhu W 2013 Chem. Soc. Rev. 422039

[6] Pramanik A, Sarkar S, Pal S and Sarkar P 2015 Phys. Lett. A 3791036

[7] Wang B, Wang Y, Hua J, Jiang Y, Huang J, Qian S et al 2011 Chem. Euro. J. 172647

[8] Qian G, Dai B, Luo M, Yu D, Zhan J, Zhang Z et al 2008 Chem. Mater. 206208

[9] Biswas S, Pramanik A, Ahmed T, Sahoo S K and Sarkar P 2016 Chem. Phys. Lett. 64923

[10] Biswas S, Pramanik A and Sarkar P 2017 Comput. Theor. Chem. $\mathbf{1 1 0 3} 38$

[11] Koumura N, Wang Z-S, Mori S, Miyashita M, Suzuki E and Hara K 2006 J. Am. Chem. Soc. 12814256

[12] Zhang X-H, Wang Z-S, Cui Y, Koumura N, Furube A and Hara K 2009 J. Phys. Chem. C 11313409

[13] Kim S, Kim D, Choi H, Kang M S, Song K, Kang S O et al 2008 Chem. Commun. (Camb.) 404951

[14] Qin H, Wenger S, Xu M, Gao F, Jing X, Wang P et al 2008 J. Am. Chem. Soc. 1309202

[15] Horiuchi T, Miura H, Sumioka K and Uchida S 2004 J. Am. Chem. Soc. 12612218

[16] Horiuchi T, Miura H and, Uchida S 2003 Chem. Commun. 24 3036

[17] Tian H, Yang X, Chen R, Pan Y, Li L, Hagfeldt A et al 2007 Chem. Commun. 363741

[18] Yang C-J, Chang Y J, Watanabe M, Hon Y-S and Chow T J 2012 J. Mater. Chem. 224040

[19] Yen Y-S, Hsu Y-C, Lin J T, Chang C-W, Hsu C-P and Yin D-J 2008 J. Phys. Chem. C 11212557

[20] Wong B M and Cordaro J G 2011 J. Phys. Chem. C 115 18333

[21] Erten-Ela S, Yilmaz M D, Icli B, Dede Y, Icli S and Akkaya E U 2008 Org. Lett. 103299

[22] Kolemen S, Cakmak Y, Erten-Ela S, Altay Y, Brendel J, Thelakkat M et al 2010 Org. Lett. 123812 
[23] Loudet A and Burgess K 2007 Chem. Rev. 1074891

[24] Ulrich G, Ziessel R and Harriman A 2008 Angew. Chem. Int. Ed. 471184

[25] Boens N, Leen V and Dehaen W 2012 Chem. Soc. Rev. 411130

[26] Baruah M, Qin W, Vallée R A, Beljonne D, Rohand T, Dehaen W et al 2005 Org. Lett. 74377

[27] Jiao C, Huang K-W and Wu J 2011 Org. Lett. 13632

[28] Rohand T, Baruah M, Qin W, Boens N and Dehaen W 2006 Chem. Commun. 3266

[29] Misra R 2017 J. Phys. Chem. C 1215731

[30] Bañuelos J 2016 Chem. Record. 16335

[31] Zhu S, Zhang J, Vegesna G, Luo F-T, Green S A and Liu H 2010 Org. Lett. 13438

[32] Hattori S, Ohkubo K, Urano Y, Sunahara H, Nagano T, Wada Y et al 2005 J. Phys. Chem. B 10915368

[33] Kolemen S, Bozdemir O A, Cakmak Y, Barin G, Erten-Ela S, Marszalek M et al 2011 Chem. Sci. 2949

[34] Ning Z, Zhang Q, Wu W, Pei H, Liu B and Tian H 2008 J. Org. Chem. 733791

[35] Ditchfield R, Hehre W J and Pople J A 1971 J. Chem. Phys. 54 724

[36] Becke A D 1993 J. Chem. Phys. 985648

[37] Ni Y, Zeng L, Kang N Y, Huang K W, Wang L, Zeng Z et al 2014 Chem. Euro. J. 202301

[38] Tomasi J, Mennucci B and Cammi R 2005 Chem. Rev. 105 2999

[39] Zarate X, Schott-Verdugo S, Rodriguez-Serrano A and Schott E 2016 J. Phys. Chem. A 1201613

[40] Sánchez-de-Armas R O, Oviedo López J, San-Miguel M A, Sanz J F, Ordejón P and Pruneda M 2010 J. Chem. Theor. Comput. 62856

[41] Andreev A S, Kuznetsov V N A and Chizhov Y V $2012 \mathrm{~J}$. Phys. Chem. C 11618139

[42] Frisch M, Trucks G, Schlegel H B, Scuseria G, Robb M, Cheeseman J et al 2009 Wallingford, CT 19227
[43] Ghosh N N, Chakraborty A, Pal S, Pramanik A and Sarkar P 2014 Phys. Chem. Chem. Phys. 1625280

[44] Barone V and Cossi M 1998 J. Phys. Chem. A 102 1995

[45] Tan Y Y, Tu W H and Manzhos S 2014 Chem. Phys. Lett. 593 14

[46] Feng J, Jiao Y, Ma W, Nazeeruddin M K, Grätzel M and Meng S 2013 J. Phys. Chem. C 1173772

[47] Lu T and Chen F 2012 J. Comput. Chem. 33580

[48] Li H-B, Zhang J, Wu Y, Jin J-L, Duan Y-A, Su Z-M et al 2014 Dyes Pigments 108106

[49] Biswas S, Pramanik A, Pal S and Sarkar P 2017 J. Phys. Chem. $C 1212574$

[50] Pratik S M and Datta A 2013 Phys. Chem. Chem. Phys. 15 18471

[51] Wei T, Sun X, Li X, Ågren H and Xie Y 2015 ACS Appl. Mater. Interf. 721956

[52] Yang Z, Liu C, Shao C, Lin C and Liu Y 2015 J. Phys. Chem. C 11921852

[53] Li M, Kou L, Diao L, Zhang Q, Li Z, Wu Q et al 2015 J. Phys. Chem. C 1199782

[54] Qu Z-W and Kroes G-J 2007 J. Phys. Chem. C 111 16808

[55] Tarsang R, Promarak V, Sudyoadsuk T, Namuangruk S, Kungwan N, Khongpracha P et al 2015 RSC Adv. 538130

[56] Monti A, Negre C F, Batista V S, Rego L G, de Groot H J and Buda F 2015 J. Phys. Chem. Lett. 62393

[57] Ding W-L, Li Q-S and Li Z-S 2015 J. Mater. Chem. A 3 19948

[58] Gupta K S, Zhang J, Marotta G, Reddy M A, Singh S P, Islam A et al 2015 Dyes Pigments 113536

[59] Ronca E, Marotta G, Pastore M and De Angelis F 2014 J. Phys. Chem. C 11816927

[60] Wei H, Luo J-W, Li S-S and Wang L-W 2016 J. Am. Chem. Soc. 1388165 